409

DOES RUGBY NEED TO ADJUST ITS THRESHOLD FOR INMATCH OFF-FIELD HEAD INJURY ASSESSMENTS (HIAS)? A RETROSPECTIVE ANALYSIS OF CONCUSSIONS DIAGNOSED POST-MATCH, WHERE NO IN-MATCH OFFFIELD HIA WAS PERFORMED

\begin{abstract}
${ }^{1,2}$ Steffan Griffin, ${ }^{3}$ Matt Cross, ${ }^{4}$ Geraint Ashton Jones, ${ }^{1,5}$ Simon Kemp, ${ }^{1,6}$ Keith Stokes. ${ }^{1}$ Rugby Football Union, London, UK; ${ }^{2}$ University of Edinburgh, Edinburgh, UK; ${ }^{3}$ Premiership Rugby Limited, London, UK; ${ }^{4}$ Alligin Performance, Glasgow, UK; ${ }^{5}$ London School of Hygiene and Tropical Medicine, London, UK; ${ }^{6}$ University of Bath, Bath, UK
\end{abstract}

\subsection{6/bjsports-2021-IOC.374}

Background Concussion is one of the key player welfare issues in rugby union. Independent Matchday Doctors (IMDDs), supported by live video feeds, were brought in to elite men's rugby union matches in England over the 2019-2020 season to support the in-match off-field head injury assessment (HIA) process.

Objectives To determine whether rugby union players diagnosed with a concussion post-match could have been removed for an in-match off-field HIA.

Design Prospective cohort study.

Setting Twelve professional rugby union clubs in the highest league in England.

Participants Professional male rugby union players.

Outcome measures 1) Number of concussions diagnosed postmatch, where no in-match off-field HIA was performed.

2) The proportion of these that were 'tagged' in-match by the IMDD on the 'Hawkeye' live video system as events with the potential to result in concussion.

Main Results Twenty-five concussions were diagnosed postmatch (21\% of total concussions). In 20 instances, an IMDD report and match-specific Hawkeye library were available for analysis. Of these, the IMDD reported a potentially significant incident in $12(60 \%)$ cases, and a potential incident was 'tagged' on Hawkeye in 13 (65\%) of cases. In 6 cases (30\%), nothing was highlighted in either the IMDD report or on Hawkeye, and in 9 cases $(45 \%)$ there was an incident highlighted on both. Common features observed in the 'tagged' video clips include: players staying on the ground after contact for longer than other players; players steadying themselves on their knees before standing; subtle gait changes; and players grasping the face after contact.

Conclusions Over 20\% of concussions were not diagnosed inmatch, and over half of these were 'tagged' as events with potential to result in concussion. Governing bodies should consider these video features when setting the threshold for HIAs. Optimising this process could help minimise the risk of negative health outcomes in players.

\section{A PROFILE OF ISOMETRIC CERVICAL STRENGTH IN ELITE PROFESSIONAL MALE RUGBY PLAYERS}

\footnotetext{
${ }^{1,3}$ Mairéad Liston, ${ }^{6}$ Darren Dahly, ${ }^{1}$ Rod McLoughlin, 2,3,4Éanna Falvey, ${ }^{3,4}$ Colm Fuller, ${ }^{5}$ Deborah Falla, ${ }^{1,7}$ Nicol van Dyk. ${ }^{1}$ Irish Rugby Football Union, Dublin, Ireland; ${ }^{2}$ World Rugby, Dublin, Ireland; ${ }^{3}$ College of Medicine and Health, University College Cork, Cork, Ireland; ${ }^{4}$ Sports Surgery Clinic Santry, Dublin, Ireland; ${ }^{5}$ School of Sport, Exercise and Rehabilitation Sciences, University of Birmingham, Birmingham, UK; ${ }^{6}$ School of Public Health, University College Cork, Cork, Ireland; ' Section Sports Medicine, Faculty of Health Sciences, University of Pretoria, Pretoria, South Africa
}

10.1136/bjsports-2021-IOC.375
Background Rugby Union, a popular full-contact sport played throughout the world, has one of the highest rates of concussion of all full-contact sports. Concussion is the most commonly reported injury in rugby matches with $17 \%$ of professional players sustaining a concussion in a given season. Cervical muscle strengthening is often included in concussion rehabilitation and/or prevention protocols however the cervical strength profiles of elite rugby players are currently unknown. Objective To describe the preseason isometric cervical strength profiles of peak strength and endurance, in elite professional male rugby players, categorised by playing position.

Design Cross-sectional study measuring isometric peak and endurance cervical strength.

Setting Elite rugby players from four professional Irish teams that compete at the highest level of competition in Ireland and Europe.

Patients (or Participants) Elite male rugby players $(n=136)$.

Interventions (or Assessment of Risk Factors) The association between isometric strength and playing position, age, height and weight was investigated.

Main Outcome Measurements Multidirectional cervical strength was measured using a standardised isometric endurance test and a novel isometric 'make' test.

Results In total, 136 players participated in this study, including front row forwards (27\%), other forwards (28\%) and backs $(45 \%)$. The average time for the endurance tests were 55.7 seconds and 52.9 seconds for extension and flexion respectively, with no statistically significant differences between playing positions.

Peak isometric strength was significantly greater in extension $(429 \mathrm{~N} \pm 104 \mathrm{~N})$ than side flexion (left, $306 \mathrm{~N} \pm 73 \mathrm{~N}$; right, $308 \pm 70)$ across all position groups; isometric flexion $(275 \mathrm{~N} \pm 65 \mathrm{~N})$ was the weakest. Age was associated with increased isometric strength.

Conclusions This study provides normative values for peak and endurance isometric strength in professional rugby players. Differences between playing positions for peak strength highlights the importance of stratifying scores in accordance to position. Pre-season testing of isometric cervical strength may aid in return to play decisions following concussion.

\section{ACUTE CONCUSSION VERSUS POST-CONCUSSION SYNDROME (PCS): HOW CAN WE PREVENT PROGRESSION?}

1,2 Sheharzad Mahmood, ${ }^{1,3}$ Ann-Marie Przyslupski, 1,2,3 Teresa DeFreitas, ${ }^{1,4}$ Martin Mrazik,

${ }^{1,2,3}$ Constance Lebrun. 'Glen Sather Sports Medicine Clinic, University of Alberta, Edmonton, Canada; ${ }^{2}$ Faculty of Medicine and Dentistry, University of Alberta, Edmonton, Canada; ${ }^{3}$ Department of Family Medicine, University of Alberta, Edmonton, Canada; ${ }^{4}$ Department of Educational Psychology, University of Alberta, Edmonton, Canada

\subsection{6/bjsports-2021-IOC.376}

Background Concussions can be classified as acute $(<90$ days to resolution) or post-concussion syndrome (PCS, $\geq 90$ days to resolution). PCS poses a great burden to the individual and to public health.

Objective To contrast the presentation and recovery of acute concussion and PCS to identify potential factors for PCS prevention.

Design Retrospective chart review of concussion patients seen by Sports and Exercise Medicine physicians from 2015-2019. Setting Glen Sather Sports Medicine Clinic, Edmonton, Alberta, Canada. 